\title{
Gacetas españolas de los Países Bajos en el siglo XVII: La Gazeta de Amsterdam y Noticias Principales y Verdaderas
}

\author{
Dr. Javier Díaz Noci \\ Facultad de Ciencias Sociales y de la Comunicación \\ Universidad del País Vasco \\ pdpdinoj@1g.ehu.es
}

\section{RESUMEN}

El periodismo antiguo, anterior a 1789, es aún hoy y a pesar de su importancia desconocido. También lo es en el caso de España y en el de las gacetas en español no sólo de la Península, sino también de los Países Bajos. Salvo, por diversas razones, La Gaceta de Madrid, los historiadores del periodismo pasan de puntillas por encima de otras gacetas producidas en el resto de España y, aún más, por encima de aquellas que se editaron en lengua española en los Países Bajos, en Flandes y Holanda, en el siglo XVII. Nosotros posamos nuestros ojos precisamente en estos periódicos, hasta ahora prácticamente desconocidos a pesar de su influencia $e$ importante papel en la época, como lo demuestra la reimpresión de varias de esas gacetas durante décadas en España, en San Sebastián concretamente. En concreto, analizamos la Gazeta de Amsterdam, publicada por la comunidad sefardí holandesa entre 1675 y 1690, las Noticias Principales y Verdaderas que aparecieron en Bruselas entre 1685 y 1704, y la reimpresión de esta última gaceta y de otro posible periódico flamenco en San Sebastián.

\section{ABSTRACT}

Early journalism -the one before 1789- is still, despite its importance, very unknown. It is so as well in the case of Spain and in the case of the Spanish-language gazettes published not only in the Peninsula but also in the Low Countries. Except, for some reasons, the Gazeta de Madrid, historians of journalism rarely take into account the other gazettes published in the rest of Spain and, moreover, those published in Spanish during the sixteenth century in Holland and Flandria. We will however speak about those newspapers, until recently almost absolutely unknown amongst us in spite of their influence and important role in that period, as can be demonstrated by the fact that some of those gazettes were published again in Spain, concretely in San Sebastián. We analyse the Gazeta de Amsterdam, published by the Dutch sephardic community between 1675 and 1690, the Noticias Principales y Verdaderas appeared in Brussels between 1685 and 1704, and the reissuing of this and another Flemish gazette in San Sebastian.

Palabras claves: Gacetas/España/Paises Bajos/Siglo XVII.

Key Words: Gazette/Spain/Low Countries/XVII Century. 


\section{Introducción}

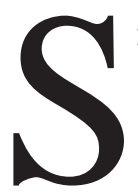

i hay un país europeo donde los estudios sobre periodismo y comunicación se han desarrollado en los últimos años, ése es España. La prueba es la bibliografía que se ha generado desde que se fundaron las primeras facultades españolas de ciencias de la información -en algunos otros sitios, de ciencias de la comunicación-, e incluso antes, ya que desde que en 1930 se crea la Escuela de Periodismo de El Debate (más tarde Escuela de Periodismo de la Iglesia) se da, más en forma de manual que de investigación, una considerable producción impresa sobre la información periodística. De entre las varias materias objeto de esta bibliografía cada vez más especializada, la historia del periodismo es una de ellas.

Se la ha denominado historia de la prensa, historia del periodismo, historia de los medios de comunicación e historia de la comunicación. Grosso modo, estamos de acuerdo en que esta rama académica todavía joven -aunque, lógicamente, cada vez lo es menos- se ha mantenido hasta la fecha dentro de un comprensible positivismo y de la hemerografía (que correspondería a la historia de la prensa), sin escorarse definitivamente hacia una perspectiva social de la producción y recepción de la información, en cuyo caso estaríamos hablando más bien de historia del periodismo o historia de la comunicación (Tresserres, 1994). El debate científico se ha producido, de forma implícita, en los países de habla inglesa, donde se ha superado ya el estadio que en 1939 describió uno de los indiscutibles padres de esta disciplina académica, Folke Dahl:

The first and most important object in the study of the history of the earliest newspaper publications is obviously of a pure bibliographical nature. (...) try to establish exactly when, where and by whom each number was printed and issued. At times the question arises whether the newspaper one is studying, is a first, second or perhaps even a third edition, or whether it is a reprint from a later date. It may even be some other contemporary publication which, for some special reason or other, pretends to be a periodical newssheet or news-book, although it is not (...). This purely bibliographical branch of newspaper research I should like to call Newspaper Bibliography (...). Newspaper are really not individuals like books, but they are more like the members of a large family (Dahl, 1939: 161).

Postura que varios académicos han abogado por trascender de forma explícita y práctica. Göran Leth (Leth, 1994), por ejemplo, siguiendo a D. H. Couvée, ha escrito en ese sentido, y sus aportaciones a la historia del periodismo (y ya no "de la prensa") se han plasmado en forma impresa en varios trabajos referidos a la prensa europea del siglo XVII, que es también el objeto de esta breve investigación nuestra. Dentro de este mismo campo tan poco trillado -al menos en 
España- que son los primeros periódicos del siglo XVII, creemos que es ejemplar, por su enfoque pluridisplinar exento de complejos y por su rigor, el estudio de Joad Raymond sobre las gacetas ("newsbooks") inglesas del período de la Guerra de Cromwell, 1641-1649 (Raymond, 1996).

Por lo que respecta a España, han sido escasos los estudios sobre lo que podríamos denominar -como se hace en el campo de la música- «periodismo antiguo», el anterior a 1789. El siglo XVII, en el que comienzan a editarse las primeras publicaciones periódicas, está prácticamente virgen de estudios. Dos son, a nuestro entender, las razones fundamentales para este vacío que comienza ya, con honrosas excepciones -la mayoría referidas a la historia de la Gaceta de Madrid-, a ser clamoroso. Una la constituyen los historiadores que se dedican a estudiar el periodismo y los medios de comunicación, la mayoría de los cuales tienen una formación en Historia Contemporánea (es decir, la referida a la era que inaugura la Revolución francesa), a cuyos departamentos pertenecen casi todos ellos. La segunda es la tremenda importancia que se da a la Gaceta de Madrid (1661) como primer y más importante periódico español. Aún aceptando que eso es cierto, en ningún modo se trata de la única gaceta publicada en suelo español ni en lengua española, dentro o fuera de nuestras fronteras, en el siglo XVII. Solventar, como se ha hecho con más frecuencia de la que sería deseable, el resto de esas gacetas como meras reimpresiones de la de Madrid es cerrar los ojos a una realidad bastante más rica de lo que se ha descrito hasta el momento. Muchas de esas primeras gacetas, entre ellas algunas que mencionaremos aquí, no deben nada a la madrileña. No se debe olvidar, por otra parte, que la propia Gaceta de Madrid no nace con las características de un periódico, título y periodicidad estables. Se hace por lo tanto acuciante un estudio sistemático y general sobre todas las gacetas españolas de ese siglo.

En cualquier caso, y por centrarnos en nuestro objetivo, vamos a dar noticia y estudiar brevemente dos que en lengua española se publicaron en los Países Bajos, tanto en el Flandes español como en la Holanda protestante y, hasta cierto punto, plural y permisiva que fue el lugar, tengámoslo en cuenta, donde se producen las primeras gacetas en francés y en inglés. Dentro de la brevedad de este artículo de investigación, vamos a intentar enfocar la cuestión desde el punto de vista de la historia del periodismo, una historia sociocultural en el sentido que a este concepto dan autores como Roger Chartier (1993 a, b y c), David Paul Nord (1990) o Peter Burke (1993), una historia del periodismo que necesariamente es una historia de la comunicación social. El periodismo (aunque en el siglo XVII la palabra ni siquiera se ha inventado) como modo de reproducción cultural, cuya investigación se desdobla y se interrelaciona en la historia de la producción y, aunque evidentemente esto resulte más difícil, de la recepción. Alguna pista de peso nos la ofrece, en uno de los casos que estudiamos a continuación, el hecho de que la gaceta bruselense a que nos referimos fue reeditada durante casi veinte años por el impresor oficial de la provincia de Guipúzcoa. 


\section{La Gazeta de Amsterdam}

\section{Cultura y comunicación en la comunidad sefardí de Holanda}

La primera, por orden cronológico, de las gacetas que en lengua española se editan en los Países Bajos es la que, desde 1675 y al menos hasta 1691, publica en Amsterdam el impresor sefardí David de Castro Tartás. Los datos los obtenemos de los ejemplares pertenecientes a las principales colecciones que conocemos, la de la Biblioteca Real de Holanda (Koninklijke Bibliotheek), varios ejemplares semanales consecutivos del año 1675 y un único ejemplar de $1690^{1}$, llamado éste Gazeta española de Amsterdam, en clara referencia a la lengua -que es el sentido en que utilizamos también la palabra en esta investigación nuestra- y no a la nación; y la que se halla en la Hemeroteca Municipal de Madrid, que comprende ejemplares de 1690 (seis números) y 1691 (siete números).

La primera noticia acerca de esta gaceta la ofrece el investigador a quien probablemente más debe la historia del periodismo en España, José Altabella. En su Evolución de los estudios de historia de la prensa en España y de la prensa como fuente histórica, no por hemerográfica y bibliográfica menos valiosa, Altabella asegura que «el primer periódico judío del mundo es una gaceta sefardí impresa en Amberes», cuyo inicio data en 1674 y del que dice estar «escrito en ladino (...) en caracteres latinos». La fuente de esos datos parece ser indirecta, en concreto un texto de 1975 (no queda claro si monografía o artículo de revista) de Maurice Kraizman, «La presse juive en Belgique et aux Pays-Bas» (Altabella, 1983: 30). A la vista de los ejemplares citados, varios datos de los mencionados por Altabella son erróneos: en primer lugar, no nos es dado asegurar con certeza que se trate del primer periódico judío del mundo. Por otra parte, aun cuando fuese impreso por un impresor de esa religión, su público parece ser más amplio -luego nos detendremos algo más en eso- que la comunidad sefardí de Amsterdam. En segundo lugar, la gaceta fue impresa en Amsterdam, y como tal se declara en el pie de imprenta de todos y cada uno de los ejemplares consultados, no en Amberes. En tercer lugar, el idioma empleado no es el ladino, sino el español de la época, y por supuesto se emplean los caracteres latinos, igual exactamente que se hacía en España. También explicaremos esto con algo más de detalle.

Los sefardíes de Amsterdam, para empezar, no provenían de los judíos expulsos de España (=Sefarad) en 1492, sino que se trataba en su mayoría de conversos o descendientes de conversos españoles y portugueses (comúnmente llamados «marranos») que, por una u otra razón, habían decidido emigrar a Holanda para abrazar de nuevo la fe de sus antepasados, el judaísmo. Se trataba, por tanto, de judíos nuevos, nacidos cristianos y educados como tales, de reciente

1 En concreto, en la colección de la Biblioteca Nacional de Amsterdam se conservan los números 1 a 53 de 1675 (faltan los números 2 y 51), que cubren desde el 7 de enero al 30 de diciembre de ese año, y el 14 de 1690, correspondiente a la semana del 3 de julio de 1690. 
implantación en los Países Bajos. Los idiomas en que hablaban eran, por tanto, el portugués y el español de la época, sin ni siquiera más rasgos dialectales judíos que los referidos al vocabulario religioso, aprendido, ya que, a diferencia de los judíos expulsados en 1492, éstos eran en España y Portugal cristianos que no vivían en grupos cerrados o en juderías que ya no existían. El idioma que se emplea en la Gazeta de Amsterdam es pura y llanamente español, no judeoespañol (también denominado «judezmo»), que se forma más tarde a partir de la evolución de la lengua española hablada por judíos lejos de España y en contacto con otras culturas y lenguas. Por supuesto, no se trata de ladino, que no es sinónimo de judeoespañol: el ladino es una lengua que jamás se ha hablado, una de las técnicamente llamadas «hagiolenguas calco», que emplean las estructuras sintácticas de una lengua «sagrada», en este caso el hebreo, en la lengua vernácula (el castellano) para traducir determinados textos religiosos. Eso es lo que se hizo a partir de la traducción de la Biblia de Ferrara, de resultas de lo cual se emplea por escrito una variedad lingüística absolutamente artificial que nunca se habló cotidianamente (Díaz-Mas, 1982, 1993: 101-102; Boer, 1996: 39).

Volvamos a la comunidad sefardí de Amsterdam. Como han demostrado recientes investigaciones de Harm den Boer, se trataba de un grupo de personas por lo general cultas, pertenecientes a la burguesía comercial, que tomaron parte activa de la vida social holandesa, hasta el punto de que produjeron una considerable, e injustamente olvidada, literatura en castellano y portugués. Y no sólo religiosa, sino también teatro y poesía laicas. En la capital de la república de las siete provincias encontraron estos nuevos judíos un lugar donde vivir y poder profesar su nueva fe en un ambiente de cierta tolerancia, desde luego muchísimo mayor que la que se les negaba en sus países de origen, España y Portugal. A partir de 1616, el municipio de Amsterdam promulgó un reglamento que permitía a los judíos practicar libremente su religión y les concedía la total ciudadanía. Los sefardíes de Amsterdam, en su mayoría judíos de pasado cristiano, se organizaron muy rápidamente, no sólo para ofrecer servicios religiosos, sino también y fundamentalmente educación, puesto que los nuevos judíos necesitaban instrucción religiosa. Entre 1600 y 1619 se fundaron tres congregaciones que se unieron en 1639 en una sola, la denominada Talmud Torá, al servicio de la cual trabajó David de Castro Tartás.

Los judíos convivían con miembros de otras religiones cristianas, la mayor parte de ellos protestantes. Algunos de ellos habían huido, como ellos, de la represión española, y venían de los territorios católicos de Flandes. También había hugonotes franceses, si bien el brazo religioso más importante de Holanda era el calvinista. Este ambiente de tolerancia (relativa, en todo caso: por influencia precisamente de los pastores calvinistas, los judíos tenían vetado cualquier tipo de proselitismo) fue, precisamente, el que provocó que Amsterdam fuese el principal foco informativo y productor de gacetas en varias lenguas de toda Europa. En 
1620-21, y por tanto antes de que apareciese en 1631 la Gazette de France de Théophraste Renaudot, un tal Jacob Jacobsz publica el Courant d'Italie \& d'Almaigne, \&c., conservado en la Kungliga Biblioteket de Estocolmo, que parece ser una versión de la famosa Courante uyt Italien, Duytslandt, \&c. que se inició en 1618 (Dahl, 1939: 195-197). Algunos años más tarde (1639-1643), Broer Jansz publica corantos y gacetas en neerlandés, inglés y francés. El multilingüismo era moneda de uso común en los Países Bajos, y el español era una de las lenguas que se utilizaban en aquellos pagos que habían sido parte del Imperio de los Austrias. La publicación de papeles noticiosos dista mucho de ser inocente o independiente, y en esta «esfera pública protestante», como la ha denominado Göran Leth, corantos y gacetas prestan un servicio ideológico muy concreto. La tesis de Leth es que, más que un movimiento religioso, el protestantismo (del que obviamente no participaban los judíos) era la expresión de una corriente europea que clamaba por la libertad nacional, intelectual, social y religiosa (Leth, 1994: 76). Lo que explica también las continuas alusiones a la casa de Orange y los Estados Generales que se hacen en la Gazeta de Amsterdam.

Las lenguas que la comunidad sefardí de Amsterdam utilizaba eran, además del holandés que a la fuerza debían de utilizar para las relaciones sociales y comerciales con el resto de sus conciudadanos, el portugués y el español de las patrias de origen de aquellos judíos ibéricos. En ningún momento, insisten los especialistas (Boer, 1996: 35), se desarrolló un dialecto especial entre ellos. Parece ser que el portugués era el idioma preferido por la comunidad en sus usos diarios, y de hecho hasta casi el siglo XIX fue el idioma «oficial» de la comunidad sefardí de Holanda. Sin embargo, para la educación y los usos cultos el idioma preferido por los judíos ibéricos, españoles o portugueses, fue el castellano. Hay que tener en cuenta que desconocían por completo el hebreo, lengua de los usos religiosos, porque la mayoría de ellos se habían educado como católicos. Por tanto, conocían el latín y, al menos los más cultos, también el francés, además de, por supuesto y cada vez más, el neerlandés. La comunidad sefardí de Holanda era, por lo tanto, capaz de publicar en seis idiomas: portugués, español, francés, neerlandés, latín y hebreo. De todas ellas, la preferida durante el siglo XVII fue el español. El hebreo se reservó para los libros sagrados y los grandes tratados religiosos. El latín se empleó para comunicarse con la comunidad académica internacional. Algunos textos, los destinados a un público más laico y local, se tradujeron al neerlandés, aunque, sobre todo en el siglo XVIII, se prefirió publicar en francés para el público de los Países Bajos. El portugués se empleó en algunos géneros literarios, aunque en éstos predominó el español, lengua que, sobre todo, se empleó para como lengua de adoctrinamiento (los libros de reeducación religiosa se escribían en español o portugués) y comunicación con la comunidad sefardí en general, no sólo la holandesa, sino también los núcleos de Hamburgo, Venecia, Londres (comunidad ésta 
formada por sefardíes provenientes de Holanda) y la cuenca mediterránea hasta Salónica. El mayor prestigio y difusión del español entre judíos y cristianos explica esa preferencia sobre el portugués.

\section{David de Castro Tartás, gacetero e impresor}

Amsterdam era un hervidero de imprentas y librerías, hasta el punto de que la «Venecia del Norte» era, a comienzos del siglo XVII, uno de los principales focos de la vida intelectual europea. Sefardíes holandeses como el hijo de españoles Baruch Spinoza, aunque más tarde renegaría de su religión, fueron parte activa de la vanguardia del pensamiento europeo. Spinoza, muerto en 1677, pudo muy bien ser uno de los lectores de la Gazeta de Amsterdam. Los cultos judíos conversos de origen ibérico necesitaban sus propios impresores. Uno de ellos, tal vez el más destacado de la época, fue David de Castro Tartás.

Es poco lo que sabemos de él, fuera de sus libros, muchos y muy buenos. Es segura su vinculación con la comunidad Talmud Torá, regida muy significativamente no por rabinos, como era habitual, sino por una junta laica y en la cual «las decisiones se tomaban más por razones económicas y políticas que por razones religiosas» (Boer, 1996: 17). La comunidad produjo una amplia literatura, religiosa o aconfesional, que va desde los sermones y los libros de instrucción a las comedias, pasando por la poesía, y se fundaron, a imitación de las que existían en España y Portugal, academias literarias. La hispanidad de la comunidad sefardí de Amsterdam era, por lo tanto, muy acusada, y su integración en la vida holandesa, a la vez, muy grande.

David de Castro Tartás pudo, por su apellido, ser un judío tanto de origen portugués como español. Es posible que, como hicieron muchos otros judíos conversos, nuestro hombre adoptara un nuevo nombre más hebreo. Eso sí, al contrario que otros sefardíes, no empleó, al menos de forma pública, un doble nombre «cristiano» y «judío» (por ejemplo, un famoso literato de la comunidad sefardí de Amsterdam firmaba unas veces Miguel de Barrios y otras Daniel Leví de Barrios), aunque sí lo hizo con sus apellidos. La utilización de más de un apellido era común entre los sefardíes: mientras uno de ellos revelaba a las claras su origen judío, otro era de abolengo hispánico-cristiano. «No debe interpretarse ni el nombre judío ni el español como un alias», advierte Harm den Boer, «ya que no había nada secreto o misterioso en ninguno de ellos: estos nombres raramente trataban de esconder la identidad de la persona en cuestión» (Boer, 1996: 45). Lo que se hacía era emplear uno u otro, o los dos, según conveniencia. Por ejemplo, cuando el sefardí en cuestión se dirigía a un público no exclusivamente judío.

Esto explica por qué David de Castro Tartás firma así alguno de los libros que salen de su imprenta y, en cambio, emplea tan sólo el segundo apellido (David de Tartás) en su gaceta. Eso mismo hizo en varios de los libros que publicó. Harm den Boer asegura que «puede explicarse este fenómeno teniendo en cuenta que la 
obra está dirigida a lectores protestantes y muy probablemente no a lectores de la península ibérica (...). Un nombre como David Tartás no es nada ibérico y es quizá por este motivo que el impresor David de Castro Tartás usara únicamente el segundo apellido en algunas ediciones, las destinadas a un público cristiano» (Boer, 1996: 45, 73). Según esto, la Gazeta de Amsterdam se dirigiría a un público no exclusivamente judío (todo aquel que en Holanda y en la Europa del momento pudiese leer en castellano, y no eran pocos quienes podían), pero no ibérico (donde, de cualquier modo, la gaceta no iba a llegar, al menos de forma legal). Está claro que esto ni siquiera se intentó: en contra de lo que era bastante común entonces, el pie de imprenta refleja exactamente la realidad. En efecto, era común que o bien no hubiese pie de imprenta o bien éste fuese falso. El propio David de Castro Tartás emplea este subterfugio en varios de sus libros, según cuál fuese su destinatario. Poner «Amsterdam» en la portada o el pie de imprenta suponía que la edición no circularía en los territorios católicos. Así que si lo que se pretendía era esto, lo habitual era hacer constar en el pie de imprenta otra ciudad menos sospechosa, por ejemplo Bruselas, capital del Flandes español, o Amberes, aunque por supuesto todos esos libros partiesen de la imprenta de De Castro en la capital holandesa.

Aunque la actividad de David de Castro Tartás está documentada desde 1663 (de ese año es la reimpresión del popular tratado Visión deleitable y sumario de todas las ciencias de Francisco de Cáceres), su actividad se intensifica precisamente por aquellos años en que comienza a editar su gaceta. Entonces es cuando la comunidad sefardí de Amsterdam se organiza mejor. Quizá la obra cumbre salida de los talleres de David de Castro Tartás son los Sermões que pregárão os doctos ingénios do kabal kadós de Talmud Torá que se imprimen en 1675, a raíz de la inauguración de la gran sinagoga de la comunidad Talmud Torá. Se trataba de una edición muy cuidada, con grabados del holandés Romein de Hooghe y prólogo del propio De Castro Tartás (Boer, 1996: 24).

No es aventurado suponer que, a partir de entonces, David de Castro Tartás se convirtiese en el impresor por excelencia de la comunidad sefardí de Amsterdam. Del establecimiento de David de Castro Tartás saldrán varios de los libros sefardíes más importantes de la época, religiosos y profanos, para judíos y para cristianos. De 1676 data la primera de las academias literarias sefardíes de la ciudad (la Academia del Temor Divino), y en 1685 se crea la Academia de los Floridos. Desde 1624 se representan comedias en español y portugués, aunque es a partir de 1667-68 cuando se organizan compañías más o menos estables, que representan sobre todo en el carnaval judío (fiesta de Purim). Varias obras del más famoso literato sefardí de la época, Miguel de Barrios, fueron dadas a la luz por nuestro impresor: Libre albedrío y armonía del cuerpo (1680), Bello Monte de Helicona (1686), como lo fueron las de otros literatos como José de la Vega y Manuel de Leão. En 1689 imprimió el Compendio de dinim de un sefardí holandés 
emigrado a Londres, David Pardo. Y de 1692 es su última obra conocida, las Ideas posibles de José de la Vega. A partir de entonces, se documenta la existencia de otro impresor, tal vez familiar directo o político suyo, un tal Manuel Texera Tartás.

En cuanto a la Gazeta de Amsterdam, se trata de un producto de segundo orden, comparado con los libros que salieron de sus prensas, pero perfectamente homologable con otras gacetas holandesas, en neerlandés, alemán, inglés, francés o italiano, salidas de las prensas de los grandes impresores de gacetas de entonces, Caspar y Jan van Hilten, Broer Jansz, Mathijs van Meininga, Joost Broersz, Francoys Lieshout o Jan Jacobsz Bouman (Dahl, 1946), y desde luego mucho mejor que cualquiera de las gacetas de otros países de la época ${ }^{2}$. La gaceta se encabeza con la mancheta Gazeta de Amsterdam, en grandes letras centradas, con un escudo en medio. A manera de subtítulo, a dos columnas, la fecha de edición. Formalmente, es desde luego un producto más cuidado que el resto de las gacetas en castellano de España o Flandes, que siempre se tiran a una sola columna. La Gazeta de Amsterdam, en cambio, se diseña a dos columnas, con titulares en mayúsculas por países (Italia, Francia, Alemania, Flandes y Holanda) y subtítulos en cursiva indicando la ciudad y la fecha (Venecia, 23 Deziembre, por ejemplo). Este diseño a doble columna era típico de las gacetas neerlandesas, y fue inaugurado en 1618 por el famoso Courante uyt Italien, Duytslandt, \&c., diseño que a su vez se tomó de las ediciones populares (protestantes) de la Biblia ${ }^{3}$. Las páginas no van numeradas en los ejemplares de 1675, pero sí, de forma correlativa con los números anteriores, a partir de 1690. En cambio, se numeran los ejemplares de cada año, cosa del todo inhabitual en las gacetas españolas, al final de la primera columna de la primera página. Eso nos permite afirmar que el primer ejemplar es el del lunes 7 de enero de 1675. Cada ejemplar semanal consta invariablemente de cuatro páginas, al término de las cuales figura el pie de imprenta: «Impresso en Casa de David Tartás» (en algún número, seguramente por error, figura la lectura «David Tartaz»). Este sencillo pero funcional diseño se mantiene hasta 1690, en que incorpora, además de la paginación ya mencionada, un encabezado de página que reza «Gazeta española de Amsterdam»

2 Los aspectos formales, tipográficos y de diseño, del periodismo histórico (y mucho menos el de esta época tan temprana a que nos referimos aquí) se han abordado muy poco. Sin duda, la tradición inglesa inaugurada por el tipógrafo y académico Stanley Morison (el mismo que diseñó el famoso tipo Times New Roman para el diario londinense The Times) es la más representativa. El propio Morison, sobre todo en su estudio de 1932 The English Newspaper, sentó las bases al analizar la evolución formal de los periódicos ingleses desde el siglo XVII al XX. El ya citado Joad Raymond (vid. Bibliografía) abordó estos aspectos en un capítulo de su tesis doctoral. En nuestro país, en cambio, todavía hay quien se niega incluso a otorgar a las gacetas, fuera de la de Madrid, el estatus mismo de periódico.

3 P. M. Handover, en su A history of the London Gazette 1665-1965 (London: Her Majesty's Stationary Office, 1965) pone de manifiesto esta relación «between bible and newspaper printing». Según él, «both pack much matter as economically as possible by using types of small size; both have long runs» (pág. 10). 
Además de las consabidas noticias internacionales, de Italia (Venecia, Nápoles, Génova, Roma, Milán), Alemania, Francia (París y Estrasburgo) e Inglaterra (Londres), lugares donde existían comunidades sefardíes, la gaceta informa puntualmente de lo ocurrido en Bruselas (lo que nos hace pensar que en esa ciudad pudo leerse con alguna asiduidad) y en Holanda. Informar del propio país no era en absoluto habitual en las naciones que, como España o Francia, estaban controladas férreamente por la censura regia. David de Castro Tartás da noticias de Amsterdam y de La Haya, las ciudades más pobladas e importantes y también las que contaban con núcleos sefardíes. La mayoría son noticias oficiales -los sefardíes estaban agradecidos por la tolerancia de la casa de Orange y los Estados Generales, y les convenía lógicamente llevarse bien con ellos- y no faltan incluso los avisos.

En cuanto a la periodicidad, depende naturalmente de la lejanía de las ciudades donde se producen las informaciones. De Italia tardan entre dos y tres semanas en llegar, entre una y dos semanas de Francia, Alemania y Londres. Sólo unos días separan la datación de las noticias de La Haya y Bruselas, cercanas a Amsterdam, y la fecha de publicación de la gaceta. Muchas de las noticias de Amsterdam son del todo frescas, y se datan el mismo día en que aparece el periódico. Éste, por lo tanto, se componía, imprimía y distribuía en un solo día, siempre los lunes. 


\section{G A P \\ DEAMS TERDAM \\ De Lunes 7. de Enero 1675 .}

\section{T A L I A.}

I'enecix =j IJezicmbre.

Jbre ciertus galtus fuperflucs

rdenò el Senado una ple:na-

ca. En el barrio llamad, de poltolos huvo Subado patra. n conciderable incendi', que $\supset$ fe huviera dilarado fino fe baran algunas calis, con que ingii) el furgo, fiendo muy Je el daño que fe ciufó: en el lo barrio fucedio la noche de es partudo ua a difgracis, y tue ripeçando tn una puente el ,eutiardo Loralono Senado- Señria. y defias mas lilutres us detita Cictíd, cayo en al y dando con la cabcça en un a dcxolavida: en la mlf:na ro. nuriò de lusiro ci Cunde $L$ u. co Vidiman, y lo milton fice11 Cavallero Aichicli à ricmpo eftava hoigando y a'egraritule fus antgos, endas eftas inuertes on muy fentilas de rnda la Noa y plebe. De MLefina le avifa movidos de amicic:on los meies de Caranes, embiusan al. ns viveres à los Rebeldes, pero e:ido avifis dello !os Realitus, Prrarng ranhier ancel mort, e ro fiendo focorticits centods

$$
\text { Nil, } 1 .
$$

la brevedad potible por los 1 cefes, le rindirar a Efpaña. Napoles 14 Driembire. Como la Corte Carholica cantas inftancias pataque rro Virrey embie algun dine Inperio y Fládes, procura th dilicuiparie diziendo haver te do diverias partidas a Sicilia ira los de Mleftina, de acionde crive haver los Rebeidles hech falida y tomado el Convento Placido y ocros pueftos rezil Scalera, plantando z.pieças . ñon en uno dellos, peru ale donie alla la Armada litia echò en rierra yoc.hombres corro de los Realiftas, que at dos con efta afiltencia, hi, retirar a los Rejeldes romar las dons pieças de Cañon: cniri havia el Marques de Bayona e in scr. hombres para recri prefidio descaleta, pero co: rando los Rebelites quanro $h_{1}$ portaltic la reltauracion de a plars, hazicn bo rodo lu esti holiveron acomererla, y tuer chejadus valerolaruente. $y$ citcn rambien tomadus roc na pesiorid le da lolanicute 5.

A 


\section{Noticias Principales y Verdaderas}

\subsection{Picas en Flandes: los gaceteros de Bruselas también hablan español}

La existencia de gacetas en español publicadas en las Provincias del Norte no debió pasar desapercibida ni para los impresores de los Países Bajos españoles ni para las autoridades de Su Majestad Católica. Algunos destacados sefardíes de Amsterdam, como el escritor Miguel de Barrios, provenían de Bruselas, donde sin duda mantenían algún contacto. Así que, aunque como hemos dicho parece claro que la Gazeta de Amsterdam jamás ocultó su origen y autores ni pretendió difundirse, al menos ostensiblemente, en Flandes, la cercanía geográfica y el idioma sin duda hicieron que el periódico fuese conocido fuera de Holanda y desde luego en los dominios españoles cercanos.

No podemos afirmar con rotundidad que fuese ésta una de las razones por las que se editó, desde al menos 1685, al menos una gaceta en español en Bruselas. Por esos años comienza a editar Pedro de Cleyn su quincenal Noticias Principales $y$ Verdaderas ${ }^{4}$. Desconocemos cuál es la fecha exacta de publicación de esta importante gaceta, ya que el primer número conservado, perteneciente a la colección de la Biblioteca Nacional de Francia, es de 1685, pero en el mismo queda claro que la gaceta lleva tiempo imprimiéndose y distribuyéndose en Flandes y España ${ }^{5}$. Muy significativamente, aquella gaceta flamenca en castellano comienza con noticias de España, traídas de la Península y reintroducidas en la misma mediante el subterfugio legal de hacerlo mediante esta gaceta en castellano que no era española y podía así escapar a la estricta censura de la monarquía. Éste era el modo, por cierto, en el que otros muchos países de Europa se permitían disponer, para desesperación de los respectivos monarcas que poco podían hacer frente a la importación legal o clandestina de noticias en la lengua de sus reinos y fabricadas en los más permisivos Países Bajos, de noticias sobre sus propios países. Aunque, sin duda, las autoridades flamencas de Su Católica Majestad Carlos II no sólo permitían, sino que probablemente también alentaban, la publicación de esta y tal

4 Pero es más que probable que existan otras gacetas flamencas en español que esperan a ser redescubiertas. Es posible que otra gaceta, de la que luego hablaremos, titulada Noticias Extraodinarias del Norte, fuese igualmente flamenca. Es obvia la necesidad que existe de un estudio de esas gacetas, algunas de las cuales sin duda se conservarán en las bibliotecas holandesas y belgas actuales.

5 Hemos localizado cuatro ejemplares de las Noticias Principales y Verdaderas, de 1689, en la Biblioteca Nacional de Madrid, y una colección compuesta por varios ejemplares correlativos de los años 1685 y 1686 en la Biblioteca Nacional de Francia. Abarca desde la gaceta impresa el 24 de julio de 1685 (noticias de 18 a 24 de julio de ese año) hasta la impresa el 2 de abril de 1686 (15 números). La colección más nutrida, 64 ejemplares de los años 1688 a 1691, se conserva en la Hemeroteca Municipal de Madrid (13 de 1688, 19 de 1689, 7 de 1690 y 25 de 1691). Intentó ser semanal, la periodicidad más ajustada en aquellos tiempos, pero acabó siendo quincenal. Se abre siempre con noticias breves de España, referidas por lo general a la familia real y su corte, y da cuenta con regularidad de noticias de Alemania, Italia, el Norte (provenientes de Hamburgo), Inglaterra, Holanda y Francia. Finalmente, en letra más pequeña, información de Bruselas, ligada a las fuentes oficiales españolas. A diferencia de las posteriores reimpresiones donostiarras, las Noticias Principales y Verdaderas de Pedro de Cleyn carecen de numeración correlativa. 
vez otras gacetas españolas en Bruselas, pudiendo así, mediante la excusa de hacer frente a las informaciones que sobre Flandes se publicaban en, por ejemplo, la Gazeta de Amsterdam, ofrecer de forma oficiosa sus propias informaciones a través de la gaceta de Cleyn. Que luego ésta se distribuyese también -necesariamente entre círculos selectos que pudiesen pagar la importación de la gaceta- en España, mediante una actitud permisiva de «hacer la vista gorda», es algo por demostrar, aunque sólo así se explica la profusión de noticias oficiales de España y Bruselas que aparecen en estas Noticias Principales y Verdaderas.

Existió al parecer una considerable producción de gacetas en lengua española en Flandes ${ }^{6}$. No hay que olvidar, por otra parte, que nuestro primer gacetero oficial, Francisco Fabro Bremundán, secretario personal de don Juan José de Austria, era igualmente flamenco. En Bruselas, capital católica de los antiguos dominios españoles en los Países Bajos, el castellano seguía siendo de uso cotidiano. El flamenco Arnaldo (Arnaud) de la Porte publicó en 1659 una gramática y un diccionario para la educación de sus compatriotas en la lengua española, en la que aseguraba que «nos está de verdad la lengua española necessaria por los infinitos negocios que se han cada día de tratar en las cortes de Madrid y de Bruselas, y por otras pláticas y estudios privados que consisten en explicar la mente de los autores españoles» (apud Alatorre, 1998: 78). Es por ello lógico que los numerosos impresores bruselenses conociesen varias lenguas.

Es relativamente poco lo que conocemos acerca del editor y primer impresor de las Noticias Principales y Verdaderas, Pierre o Pedro de Cleyn (pues ambos nombres empleó). La mayoría de los datos provienen del Dictionnaire des Journaux (1600-1789) de Jean Sgard (Sgard, 1991: 1079). Durante un tiempo aparece como impresor probable y vendedor seguro de las Relations Véritables, una longeva gaceta (se publicó entre 1652 y 1741) en lengua francesa impresa en Bruselas. Aparece en ella como Pierre de Cleyn ${ }^{7}$. Era, en principio, semanal, aunque más tarde se convierte en bisemanal. Cleyn no fue el primer impresor de este periódico. Comenzó su publicación el impresor Guillaume Scheybels, y se vendía en casa de Guillaume Hacquebaud, cerca de la escuela de los jesuitas de Bruselas. En 1666 Scheybels se asocia a Cleyn, y poco después, entre enero y mayo de 1667, deja de aparecer el periódico. Tras esta crisis Pierre de Cleyn figura como único impresor, hasta que en 1685 (se publica ya la gaceta Noticias Principales y Verdaderas), en agosto concretamente, pasa a figurar como impresor Gilles Stryckwant, aunque el establecimiento de Cleyn figura como lugar de venta,

6 Que sepamos, no existe demasiada bibliografía al respecto. José Altabella, sin embargo, cita en su tesis doctoral dos referencias, que no queda claro si son monografías o artículos de revista: Luykx, Theo (1964): De eerste gazettiers en hun kranten in de Spaanse Nederlanden. En Hendelingen XVIII. Ondenaarde: Sanderus y Luykx, Theo (1965). Brugse krante en gazettiers in de Spaanse Nederlanden. Brugge. Ninguna de las dos figura en los catálogos de las principales bibliotecas holandesas y belgas. 
probablemente porque Cleyn (¿tal vez por indicación de los ministros de Carlos II en Flandes?) se dedica sólo a editar e imprimir la gaceta española ${ }^{8}$. Las Relations Véritables defienden a lo largo de toda su existencia la causa católica frente al protestantismo, por lo que tanto esta publicación en francés como la gaceta en castellano de Cleyn, además de disfrutar de privilegio como consta en el colofón, disponían de fuentes de información oficiales. Bastantes números de las Relations comienzan con noticias de Madrid, por ejemplo, noticias que por supuesto tienen carácter oficial y hablan del rey y de la Corte.

Se sabe que Pierre de Cleyn falleció en agosto de 1690, ya que las Relations y las Noticias Principales y Verdaderas continúan vendiéndose en su establecimiento frente a las escuelas de los jesuitas de Bruselas, ahora denominado «chez la Veuve de Pierre de Cleyn». Ambas gacetas continúan publicándose tras su muerte: todavía en 1696 y 1704 Pedro de Huarte reimprime los números más recientes de las Noticias Principales y Verdaderas en San Sebastián, y en Bruselas toma el cargo de imprimir el periódico, a partir de noviembre de 1691 Pedro Dobbeleer, junto al mercado de la Leña.

Como ya hemos dicho, esta gaceta de Cleyn es una de las pocas de la época escritas en castellano que ofrece información sobre España. Las noticias sobre el reino sufrían un curioso proceso de ida y vuelta que permitía así eludir la legislación restrictiva de Felipe IV, todavía vigente. En el primer número citado se dice: «Haviendose quexado de España de ser las noticias esteriles, por no publicarse sino cada 15 dias, y suceder continuas novedades, se ofrecen todos los ocho, con permission, haviendo empeçado à 18 del corriente, para que se satisfaga à la curiosidad Española». Las reimpresiones donostiarras de las que a continuación hablaremos eliminarán cualquier referencia a la información española, por otra parte poco peligrosa para la monarquía borbónica, ya que está claro que las fuentes de información manejadas por la oficina bruselense de Cleyn son oficiales: en todo momento se recalca en las gacetas originales que se cuenta «con permission».

Algo sabemos del modo en que las gacetas en que participó P. de Cleyn funcionaban. Entonces como hoy, la base del prestigio de un periódico era una buena información internacional, de primera mano, por tanto muy cara. La otra gaceta en que participó, Relations Véritables, se componía de boletines enviados por los corresponsales destacados en las capitales de Europa: Roma, Venecia, Génova y Milán en Italia, Hamburgo como principal suministrador de las noticias del Norte, Londres, París y Viena. También, lo que, como ya hemos dicho, resulta significativo, de Madrid. La mayoría de las noticias la componen las informacio-

8 Los pies de imprenta de los números de esta gaceta flamenca en francés consultados en la Hemeroteca Municipal de Madrid, correspondientes a 10 números de 1688 y 19 de 1691, indican que «se vendent chez Pierre de Cleyn», o, ya muerto éste, «chez la Veuve de Pierre de Cleyn». Según el propio pie de imprenta, cabe más bien colegir que el impresor, o el editor, era Gilles Stryckwant. 
nes diplomáticas y militares, en especial las referidas a batallas. Esas mismas informaciones engrosaron, traducidas y adaptadas al castellano y destinadas al mercado español, las Noticias Principales y Verdaderas. Una comparación entre números cercanos de las Relations Véritables y las Noticias Principales y Verdaderas nos permite corroborar este aserto. La gaceta en castellano se imprimía un día antes, y era más breve (cuatro páginas) que la gaceta en francés, que generalmente constaba de ocho. No se repetían necesariamente en una y otra las noticias, aunque sí ocurre en buena medida con las de España, lo que por supuesto nos hablaría de un origen común. En el último número conocido de las Noticias Principales y Verdaderas de Cleyn se lee: «El limitado despacho de estas Noticias, y las pocas assistencias de quien las recoge, sufocan los grandes desseos que tiene de satisfazer à la curiosidad publica, como tambien le impossibilitan à sacar a luz muchas obras, en que incessantemente se fatiga, no obstante sus achaques, y edad, entre ellas la Recopilacion historica de los sucessos y guerras de Ungria, desde su principio, hasta el año presente». Para fortalecer el negocio, tanto Cleyn como Stryckwant intentaron ofrecer numerosas noticias de Bruselas que añadir a los boletines internacionales que adquirían en el mercado flamenco.

¿Cuáles son los temas de estas Noticias Principales y Verdaderas? Fundamentalmente son de tipo político y militar $-\gg$ Los progresos de la Liga Sagrada de este año de 1687", «La Coronación del Augustissimo Archiduque Joseph de Rey de Ungria», expediciones ordenadas por la Corona inglesa «contra los piratas del mar del sur», etc.- y no faltan tampoco las relativas a los aconteceres, a veces anecdóticos, de la Corte Vaticana («Baxando del Monte Quirinal con su carroça el Cardenal de Estrées se vido en grandissimo peligro de la vida, por haverse rompido el freno de uno de los cavallos»), de la nobleza toscana («A los principios de este año llegò à Milàn, aunque incognito, el Gran Principe de Toscana Ferdinando de Medicis»), todas ellas correspondientes al ejemplar del 16 de febrero de 1688. No obstante, las principales noticias, como decimos, eran bélicas y políticas, referidas a los países de la Liga Sagrada (de hecho, «Progresos de los de la Liga Sagrada» es el encabezamiento de la mayoría de los números de esta gaceta). Sólo más tarde, ya en 1696, se incorporarán noticias de los países nórdicos, noticias fechadas en «Stockholm» $\mathrm{y}$ «Coppenhaguen», donde se producían serias diferencias entre la Corona (Cristián V) y el Duque de Hostein. Las noticias foráneas eran, como es lógico debido al estricto control gubernamental en cada uno de los países, mejor conocidas en el extranjero que en el ámbito propio.

En cuanto al diseño, es bastante más menguado que el de sus vecinas gacetas holandesas. La mancheta encabeza la primera de las cuatro páginas en cuarto de que consta el periódico. A modo de subtítulo, como es costumbre en la época, la fecha de las noticias: «Desde 18 hasta 24 de Julio de $1685 »$, por ejemplo. El texto se dispone a una única columna, la primera letra es capital. Primero, como es de suponer, las noticias de España (seguramente condición sine qua non 
impuesta por la monarquía española para permitir la publicación de la gaceta), siempre de carácter oficial. A continuación, noticias de Italia, Alemania, «del Norte» (Hamburgo, Polonia, Moscú, etc.), Inglaterra, Holanda y Francia, muy breves siempre. Cada una de ellas, sin fecha, está encabezada por un «De [país de que se trata]» en versalitas. Buena parte de la última página se destina a las noticias de Bruselas (que seguramente eran las que realmente atraían a los compradores flamencos, y permitían hacer algo de dinero a Cleyn), generalmente en un cuerpo de letra más pequeño que el resto, y finalmente el pie de página: «En Brusselas [fecha]». A partir de 1689 se indica «con licencia» y que «hallanse en casa de Pedro de Cleyn, frente de las Escuelas de la Compañía de Jesus» (y, ocasionalmente, «en casa de Francisco Foppens»). 


\section{$N I I C I A S$ \\ P I I EIPALES \\ $\checkmark$ VERADERAS. \\ Diffe to bafta 24 . de Fullo 1685 s.}

D España. Con el avifo que turo Su Magertad de la enfermedad peligrofa del Serior Marnues de Gra. $\mathrm{na}$, y por las inftancias con que pedia fu licencia, $\mathrm{cm}$; blo orden al señor Duque de villahermofa, paraque fe dif. pufiefle à partir con toda brevedad para trenir à governar fegunda rez eftos Éftados. Ha hecho merced del Tufon de oro al Duque de Aríchot Capitandela noble guardia de las Atcheros, antes de partir para el Pais-Baxo, haviendo llegado defta Vílla de Bruffelas en 17 . del corriente, como fe 4a dicho en las noticias antecedentes.

En 7. de Mayo faliò à campaña Don Juan Antonio Paniagua Marq̨ues de Santa Cruz, Governador de Oran con 1300. Infantes, y 400. Cavallos, dos pieças de artilleria y 3000 . Moros de paz quellevavan la vanguardia, contra multitud innumerable de Turcos, Moros, y Alurbes, $y$ al primer rencuentro fueron derrotados los Moros de paz. retirandofe en confufion; peto avançando nueftra Cavalley ria y Infantéria, ataco con tanto valor ì los Barbaros, erị numero mayor de treinta por uno de los nueftros, que los obligarón a tomar la fuga muy defordenada, con perdida de muchiffimos de ellos, y algunos prifioneros, quedando. poinuifimos de los Efpañoles, y librandofe aquella llave do Is Chrifiandad, mas por affiftencia Divina, que por humanas fuerças , de las amenaças de los Mahometanos, aunque affiftidos con Ingenicros y foldados, que dizen profecan la fec Catholica; y fe impufo filencio à la maliciofa embiia que la publicara por perdida. 


\subsection{La recepción de las gacetas flamencas en España: las gacetas donostiarras de los Huarte}

Las gacetas flamencas en español se conocían en España. Ocurrió con las Noticias Principales y Verdaderas, que se reimprimieron durante aproximadamente veinte años en San Sebastián. La gaceta que fundó Pedro de Cleyn tuvo, a pesar de sus dificultades, una vida longeva, ya que en 1704 se reimprimió en San Sebastián el último de sus números conocidos.

Desde 1687, puntualmente cada quince días, la gaceta sale de la imprenta, situada en los aledaños de la iglesia de San Vicente en San Sebastián, de la familia Huarte (los hermanos Pedro y Bernardo, junto con su madre María de Aculodi, aunque ésta probablemente figure en los pies de imprenta más como manera de conservar una fuente de ingresos como viuda del primer impresor de la Provincia), impresores oficiales de Guipúzcoa y sucesores de Martín de Huarte, el primer impresor estable que puso sus talleres en dicho territorio. Como la periodicidad (y al parecer la demanda del público, sediento de noticias en todo el Continente) más habitual era la semanal, Huarte decidió editar, en las semanas en que no aparecía la reimpresión de las Noticias Principales y Verdaderas, otra gaceta, de origen desconocido, aunque tal vez fuese igualmente flamenco: Noticias Extraordinarias del Norte. Otras alternativas, aunque más improbables, son igualmente posibles: en primer lugar, la más remota es que fuese una gaceta de gacetas compuesta por el propio Huarte. Nos inclinamos a pensar, sin embargo, que reimprimía una gaceta y actuaba así como impresor y editor, no como periodista. Otra posibilidad es que la gaceta que Huarte reimprime sea a su vez la adaptación al castellano de una o varias gacetas y corantos en lengua francesa. Sin embargo, hay que descartar que la gaceta fuese la traducción de otra gaceta francesa. Aunque es prácticamente seguro que Pedro de Huarte conocía el idioma -había perfeccionado los conocimientos del oficio de impresor aprendidos de su padre en Burdeos- no parece posible que se tradujese con tan sólo unos días cada semana tantas noticias, y tampoco aparece en la redacción rastro alguno de galicismo. Lo más probable, repetimos, es que existiese otra gaceta en español proveniente de los Países Bajos, donde el tráfico informativo era constante y las noticias se compraban y vendían como cualquier otra mercancía.

Sea como fuere, ambas gacetas se imprimían y vendían en semanas alternas, de manera que durante varias décadas San Sebastián contó con información semanal, aunque fuese a través de dos títulos diferentes. Como resulta obvio, los Huarte pretendían así servir a los intereses de su comunidad, de manera que reimprimían dos periódicos cuya información era complementaria, de resultas de lo cual San Sebastián fue una de las capitales mejor informadas de los reinos de España: ninguna ciudad contó con la presencia ininterrumpida durante tanto tiempo de dos gacetas que, según sabemos, no faltaron a su cita semanal. A nosotros no han llegado todos los ejemplares salidos de las prensas de los Huarte. Una 
colección está más nutrida que otra: la de las Noticias Principales y Verdaderas que se ha conservado llega hasta 1696, aunque sabemos que en manos privadas hay un ejemplar de 1704, lo que nos permite saber también de la longevidad de aquella gaceta flamenca original; la de Noticias Extraordinarias del Norte sólo hasta 1689 , sin que sepamos si se prolongó más allá9.

¿Por qué decidió Pedro de Huarte editar durante tanto tiempo varias gacetas, siendo además pionero en hacerlo en prácticamente todo el Norte de la Península, en una ciudad que entonces, a pesar de su importancia como puerto de mar, centro comercial y su cercanía a la frontera con Francia, no dejaba de ser un núcleo de población pequeño? Las razones son dos: una, la inquietud de la burguesía comercial, que necesitaba conocer las noticias de lo que acontecía en Europa, y que en 1727 fundará la Compañía Guipuzcoana de Caracas (aquel año, Huarte edita un semanario, ahora original, titulado Extracto de Noticias Universales). Y segunda, los Huarte, como antes Jaume Romeu y como su coetáneo Rafael Figueró en Barcelona, necesitaban «garantizar la marcha de su negocio en una época en la que, siendo los libros un objeto de lujo cuyas ventas se reducían drásticamente en las épocas de crisis, era más razonable buscar la manera de asegurarse unos ingresos modestos, pero seguros e inmediatos, a través de la producción de unos impresos breves (de cuatro hojas en $4^{\circ}$ ) que, a diferencia de los libros, eran susceptibles de una difusión amplia y rápida» (Reula Biescas, 1993).

9 Ambas colecciones, en realidad una sola y como tal encuadernada en un solo tomo, se guardan en la Hemeroteca Municipal de Madrid. 


\section{Nu: 18. NOTICIAS PRINCIPALES, Y VERDAEERAS Defde 2. alta 36. de Agolto de 1;04. DE L NOR T E.}

$\mathrm{D}$ E Varforia 7ulio 17. A 12. de cftemcs, diacnque por inflanciss del Rcy ec jut.

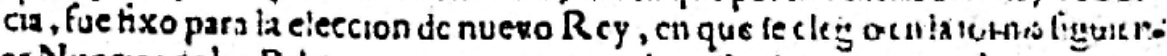
te. Los Nuncios de los Palazinos, concurrieron al pucllo donce ic cetwis hazer at tict -

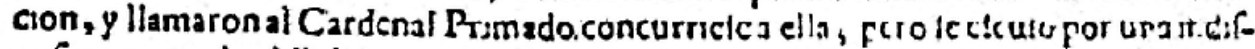

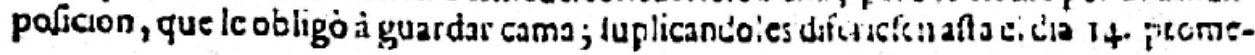

jes no ava perfom alguna ece conficeracion, que vigunos fucron calliga.0s.-El Conde de Tolofis, tuvo avilo, de que ia A rmida cnemig tofta azls Mslaga , y scria hazerve. la el mifmo via 2. enfuburca.

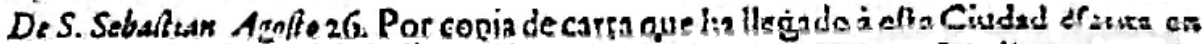

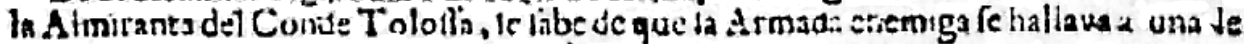
gua de diftancia ariajiendo, que en hreve fe puede oyr la accionde un lungr:ento combste

\section{Impreffas martes 26. de Aggfo de 1704 . \\ Por PEDRO de UGARTE, Imprefior de elta muy Noble, y muy Leal Ciudad de SAN SEBASTIAN.}

En un artículo publicado en La Voz de España 1949 José María Donosty afirmaba haber consultado un ejemplar de Noticias principales y verdaderas impreso en San Sebastián a finales del mes de agosto de 1704. En ese mismo artículo, Donosty incluía la reproducción de sendos fragmentos, el encabezamiento y primeras líneas, por una parte, y las líneas finales y el pie de imprenta, por otra, de aquel ejemplar, entonces en manos privadas y hoy en paradero desconocido. Los fragmentos citados son los que hemos reproducido aquí.

\section{Conclusiones}

Las gacetas estudiadas, como todas las de la época, no son productos espontáneos, sino que responden a las necesidades de determinados grupos sociales (los sefardíes en el caso de Amsterdam, las elites funcionariales en el caso de las de Bruselas, la burguesía comercial donostiarra en el caso de las reimpresiones de los Huarte), que a su vez se proyectan a otros grupos sociales e incluso se internacionalizan. Es obvio que estas publicaciones cumplen una función de reproducción de las ideologías dominantes, que las alientan o toleran. La mano de la monarquía española del último de los Austrias, preocupada por imponer sus puntos de vista en sus dominios de los Países Bajos, es notoria en el caso de las Noticias Principales y Verdaderas, y la publicación de avisos oficiales de los Estados Generales de Holanda en la Gazeta de Amsterdam nos habla bien a las 
claras de hasta qué punto la casa de Orange veía con buenos ojos la gaceta de los sefardíes holandeses.

Puesto que era impensable un periodismo local o nacional a la usanza actual, se desarrolló un periodismo de contenido internacional, basado en la crónica noticiosa de hechos, «un tiempo en que relato histórico y relato periodístico apenas tienen diferencia», como señala acertadamente Aguilera Castillo (1989: 45), que permitía una mayor libertad de información siempre dentro de un estricto carácter oficialista más que oficial.

Algunas afirmaciones que se han vertido acerca del periodismo antiguo español debieran ser en nuestra opinión revisadas. En primer lugar, la especie de que la Gaceta de Madrid (y sus reimpresiones de provincias) fue hasta bien entrado el siglo XVIII prácticamente el único periódico español, y por tanto la única fuente de información oficial u oficiosa, se tambalea. Existían otras gacetas no sólo en Holanda (ésta, si se quiere, un caso aparte, por ser el portavoz, en principio, de una comunidad que ya no estaba compuesta por súbditos de la monarquía hispánica) o en Flandes, sino que provincias periféricas como Guipúzcoa conocían y reeditaban puntualmente gacetas españolas foráneas, lo que hace que los habitantes que podían costearse esas gacetas estuviesen tan bien o mejor informados que los que residían en la Corte, sin depender de la Gaceta de Madrid.

Otra afirmación muy extendida entre la comunidad científica debe ser igualmente revisada: aquella que asegura que los Países Bajos, en concreto Holanda, eran productores de información para toda Europa, y que ésta se producía, además de en neerlandés, en francés o inglés (y también en español, como se ha visto), por lo que era por tanto no el único, pero sí principal foco informativo de todo el Continente. La alternativa a ese foco informativo protestante que escapaba a la censura tenía lugar en el Flandes español, en el propio idioma del maltrecho imperio formado por Carlos $\mathrm{V}$ y en cuyos dominios se ponía ya el sol.

En definitiva, el estudio general y profundo de los primeros periódicos en español, dentro o fuera de lo que entonces eran los territorios de la monarquía hispánica, y de toda la dimensión que encierran sus múltiples aspectos, es aún un empeño sin realizar. Ni siquiera disponemos de un catálogo de todos los títulos, de sus características morfológicas y de su localización. Si va a acometerse o no, es algo que no nos toca a nosotros decir. Sólo hemos querido que estas líneas reflejen y sirvan de muestra, aunque sólo sea en parte, de esa desconocida riqueza del periodismo antiguo español. La mano diestra y paciente de los historiadores debe, como si se tratara de un cuadro de la época, cubierto por la pátina del tiempo, el polvo, el olvido y la desidia de siglos, restaurar el vasto lienzo y descubrir ahora todos sus colores y matices. 


\section{Bibliografía:}

- AGUILERA CASTILLO, César (1989): «Historia y periodismo en el tiempo de los Mercurios». En: Revista de Ciencias de la Información. Madrid: Universidad Complutense, págs. 25-48.

- ALATORRE, Antonio (1998): El apogeo del castellano. México; Madrid: Fondo de Cultura económica.

- Altabella, José (1983): Evolución de los estudios de historia de la prensa en España y de la prensa como fuente histórica. Madrid: Editorial de la Universidad Complutense.

- BOER, Harm den (1996): La literatura sefardí de Amsterdam. Alcalá de Henares: Instituto Internacional de Estudios Sefardíes y Andalusíes de la Universidad de Alcalá.

- BURKE, Peter (1993): «La nueva historia socio-cultural». En: Historia Social. Otoño de 1993. Valencia: Instituto de Historia Social de la U.N.E.D.

- CHARTIER, Roger (1993a): «De la historia social de la cultura a la historia cultural de lo social». En: Historia Social. Otoño de 1993. Valencia: Instituto de Historia Social de la U.N.E.D.

- CHARTIER, Roger (1993b): «Las líneas de la historia social». En: Historia Social. Otoño de 1993. Valencia: Instituto de Historia Social de la U.N.E.D.

- DAHL, Folke (1939): «Amsterdam. Earlier Newspaper Centre of Western Europe. New contributions to the history of the first Dutch and French corantos». En: Het Boek, XXV, 3. The Hague: Martinus Mijhoff, págs. 161-198.

- DAHL, Folke (1946): Dutch corantos 1618-1650. A bibliography. Göteborg.

- DAHL, Folke (ed.) (1960): The Birth of the European Press. Stockholm: The Royal Library.

- DÍAZ-MAS, Paloma (1982, 1993): Los sefardíes. Historia, lengua y cultura. Barcelona: Riopiedras.

- DÍAZ-MAS, Paloma (dir.) (1987): Los sefardíes: cultura y literatura. San Sebastián: Servicio Editorial Universidad del País Vasco.

- DÍAZ NOCI, Javier (1994): «Los inicios de la prensa vasca: primeros pasos y formas protoperiodísticas». En: Revista Internacional de los Estudios Vascos. Año 42. Tomo XXXIX. N 2 (1994), p. 245-275.

- GÓMEZ MOMPART. Josep Lluís (coordinador) (1996): Metodologías para la historia de la comunicación social. I Encuentro de la Asociación de Historiadores de la Comunicación, Bellaterra, 6 de octubre de 1995. Barcelona: Servei de Publicacions de la Universitat Autònoma.

- LETH, Göran (1994): «A protestant public sphere: The early European newspaper press». En: Studies in newspaper and periodical history 1993 Annual. Westport, Connecticut: Greenwood Press, págs. 67-90.

- MORISON, Stanley (1932): The English Newspaper. London. 
- NORD, David Paul (1990): "Intellectual History, Social History, Cultural History... and Our History". En: Journalism History, Vol. 67, n 4, Winter 1990, p. 645-648.

- RAYMOND, Joad (1996): The invention of the newspaper. English Newsbooks 1641-1649. Oxford: Clarendon Press.

- REULA BIESCAS, Jaime (1993): "Una aproximación a la 'prensa' catalana del siglo XVII a través de la colección de los folletos Bonsoms". En: Treballs de Comunicació, 4, octubre. Barcelona: Societat Catalana de Comunicació.

- SGARD, Jean (dir.) (1991): Dictionnaire des Journaux (1600-1789). Paris; Oxford: Universitas; Voltaire Foundation.

- TRESSERRES I GAJU, Joan Manuel (1994): "Història de la premsa, història del periodismo, història de la comunicaciò". En: Gazeta. Actes de les primeres jornades d'història de la premsa. Barcelona: Societat Catalana de Comunicació, págs. 67-78.

(Recibido el 10-12-2001, aceptado el 15-2-2002) 